\title{
Atresia de coana bilateral en adulto con malformación craneofacial asociada: un diagnóstico poco habitual
}

\section{Bilateral choanal atresia in adult with associated craneofacial malformation: an unusual diagnosis}

\author{
Jesús Gimeno H. ${ }^{1,3}$, Almudena Alonso O. ${ }^{2}$, Pablo Sarrió S. ${ }^{1}$, \\ Manuel Gómez S. ${ }^{1,3}$, María Cruz Iglesias M. ${ }^{1,3}$
}

\section{Resumen}

La atresia de coana es una rara malformación congénita improbable de encontrar de forma bilateral en un adolescente o adulto. Hasta la fecha, no se ha descrito ningún caso de atresia bilateral en un adulto con una malformación asociada de cabeza y cuello que haya requerido tratamiento conjunto. El tratamiento de elección de la atresia de coana bilateral continúa siendo la cirugía endoscópica, con controversia en el uso intraoperatorio de mitomicina o la colocación de stents para evitar estenosis. Lo que no está claro es el orden de tratamiento y la simultaneidad del procedimiento si se asocian otras posibles patologías que tengan una indicación quirúrgica. Presentamos un caso clínico que cumple con todos estos requisitos.

Palabras clave: atresia de coana, bilateral, adulto, malformación craneofacial.

\begin{abstract}
Choanal atresia is a rare congenital malformation that is unlikely to be found bilaterally and is seldom diagnosed in adulthood. To date, no clinical case of bilateral atresia has been described with a head and neck malformation that requires surgical treatment in an adult. The preferred treatment is still endoscopic sinonasal surgery with discrepancies of the use or not of intraoperative topical mitomycin or the placement of stents to avoid restenosis. What is not clear is the order of treatment and simultaneity of the procedure with other possible associated pathologies that have a surgical indication. We present a clinical case that meets all these requirements. Keywords: choanal atresia, bilateral, adult, craneofacial malformation.
\end{abstract}

\author{
'Servicio de Otorrinolaringología. \\ Hospital Clínico San Carlos. Madrid, \\ España. \\ ${ }^{2}$ Servicio de Cirugía Maxilofacial. \\ Hospital Clínico San Carlos. Madrid, \\ España. \\ 32Departamento de Inmunología, \\ Oftalmología y Otorrinolaringología. \\ Facultad de Medicina, Universidad \\ Complutense. Madrid, España. \\ Los autores declaran no tener \\ conflictos de interés. \\ Recibido el 8 de noviembre de 2020. \\ Aceptado el 23 de enero de 2021. \\ Correspondencia: \\ Jesús Gimeno H. \\ Hospital Clínico San Carlos. \\ Otorrinolaringología. \\ Profesor Martín Lagos s/n; CP: 28040 \\ Madrid. España. \\ Email: j-gimeno@hotmail.com
}

\section{Caso Clínico}

Paciente nacida por parto eutócico con bajo peso y rasgos dismórficos faciales (cráneo normoconfigurado, microftalmia derecha, labio leporino izquierdo con hipoplasia maxilar izquierda, frenillo labial superior lateralizado y paladar ojival íntegro), por lo que ingresó en UCI neonatal hemodinámicamente estable, pero con respiración ruidosa, tiraje intercostal y supraesternal sin polipnea. Se descartó síndrome congénito malformativo asociado.
Fue intervenida a los 6 meses de edad de labio leporino y en varias ocasiones de deformidad labial superior. Ceguera de ojo derecho y alteración de la agudeza visual en ojo izquierdo. Derivada a otorrinolaringología a los 15 años, por presentar rinorrea bilateral y respiración oral crónica; como antecedentes de interés para la cirugía además de las intervenciones previas por su labio leporino, presenta una disminución de la agudeza visual en el ojo izquierdo del $60 \%$, y una ceguera completa en el ojo derecho. En la exploración con fibroscopio flexible 
Figura 1. Imagen preoperatoria, a: fibroscopio flexible; secreciones retenidas en fosa nasal derecha; b: fibroscopia flexible: secreciones retenidas en fosa nasal izquierda; c: TC corte axial: desviación septal obstructiva izquierda con hipoplasia de seno maxilar izquierdo y atresia ósea de coana bilateral junto con retención de secreciones en ambas fosas nasales; $\mathbf{d}$ : TC corte coronal: asimetría septal posterior, hipoplasia maxilar izquierda y retención de secreciones en fosas nasales.

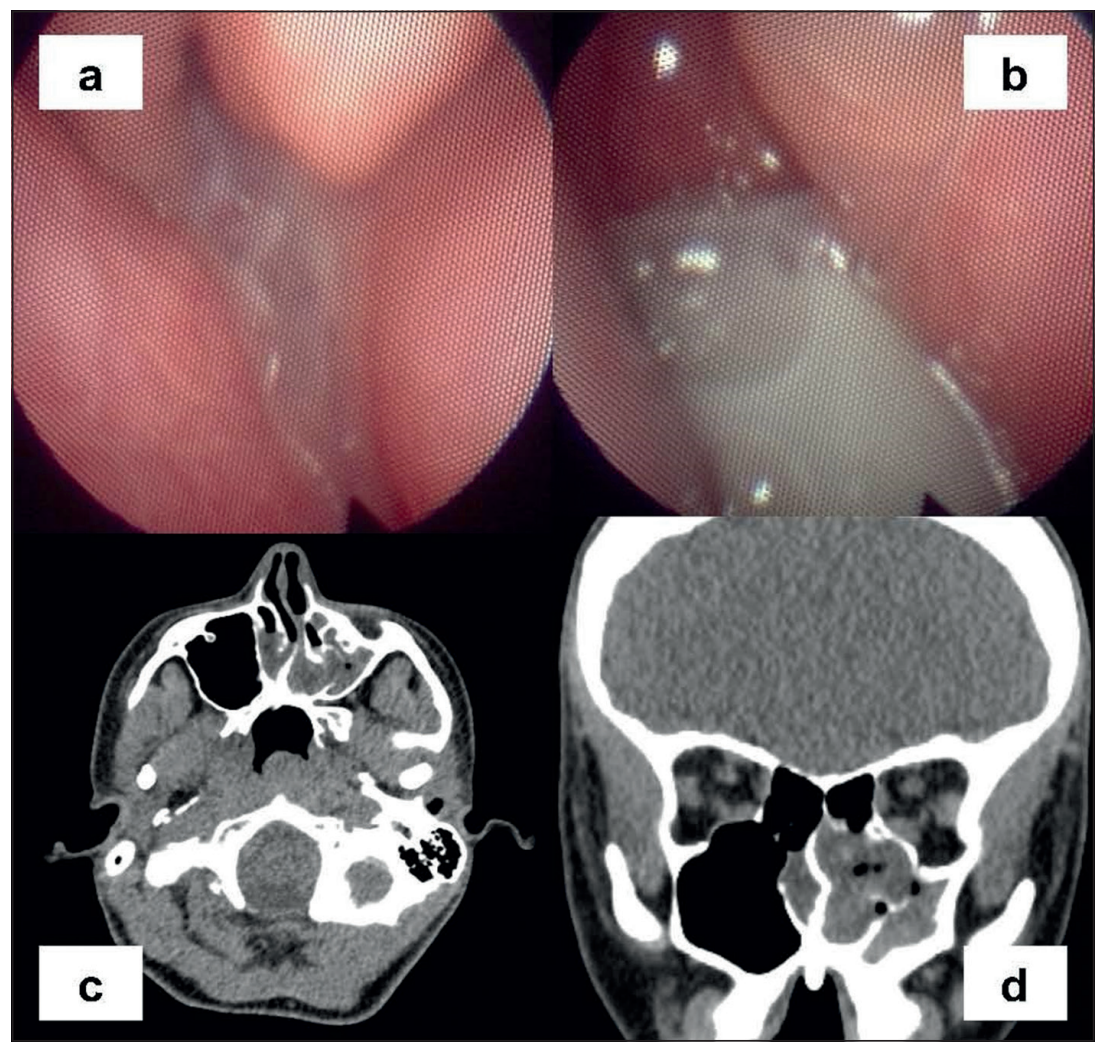

(Figura $1 \mathrm{a}, \mathrm{b}$ ) se aprecia tras aspiración de moco retenido en fosa nasal una deviación septal severa hacia la izquierda con imperforación de coana bilateral y una asimetría en el suelo de la fosa nasal derecha (más alto) con respecto a la izquierda. Se solicitó una tomografía computarizada (TC) de senos paranasales (Figura $1 \mathrm{c}, \mathrm{d}$ ) que confirmó el diagnóstico (atresia de coana ósea bilateral, hipoplasia de seno maxilar izquierdo y desviación septal).

La intervención, consistente en una cirugía endoscópica nasosinusal (CENS - septoplastia), no se realizó hasta los 19 años de edad, momento en el que terminó el tratamiento ortopédico-ortodóncico de su compresión maxilar. Se realizó una septoplastia convencional y una meatotomía media izquierda para drenaje de seno maxilar y una coanoplastia por vía endoscópica con técnica de colgajo cruzado bilateral. Se utilizó mitomicina C tópica y estabilización de los colgajos mediante cola de fibrina y hemostático reabsorbible. No se realizó sondaje nasal y sólo se utilizó unas láminas de silicona para la estabilización de los túneles mucosos de la septoplastia. No hubo complicaciones posquirúrgicas inmediatas y el posoperatorio cursó sin incidencias, pero en el período de seguimiento del mes $3^{\circ}$ al $6^{\circ}$ se objetivó una perforación septal sin clínica asociada. 3 años después de la cirugía la neocoana está permeable (Figura 2).

\section{Discusión}

La atresia de coana es una malformación congénita rara, descrita por primera vez por Roderer in 1755. Con una incidencia de 1/8.000 recién nacidos vivos, la mayoría de las veces es unilateral, siendo los casos bilaterales mucho menos frecuentes, describiéndose por primera vez en 1991 en un paciente de 16 años ${ }^{1}$. Posteriormente se describieron más casos, Canovas-Llorens y cols. ${ }^{2}$ en 1994 o Latifi y cols. ${ }^{3}$ en 1999. Puede presentarse aislada o asociarse a síndromes malformativos 


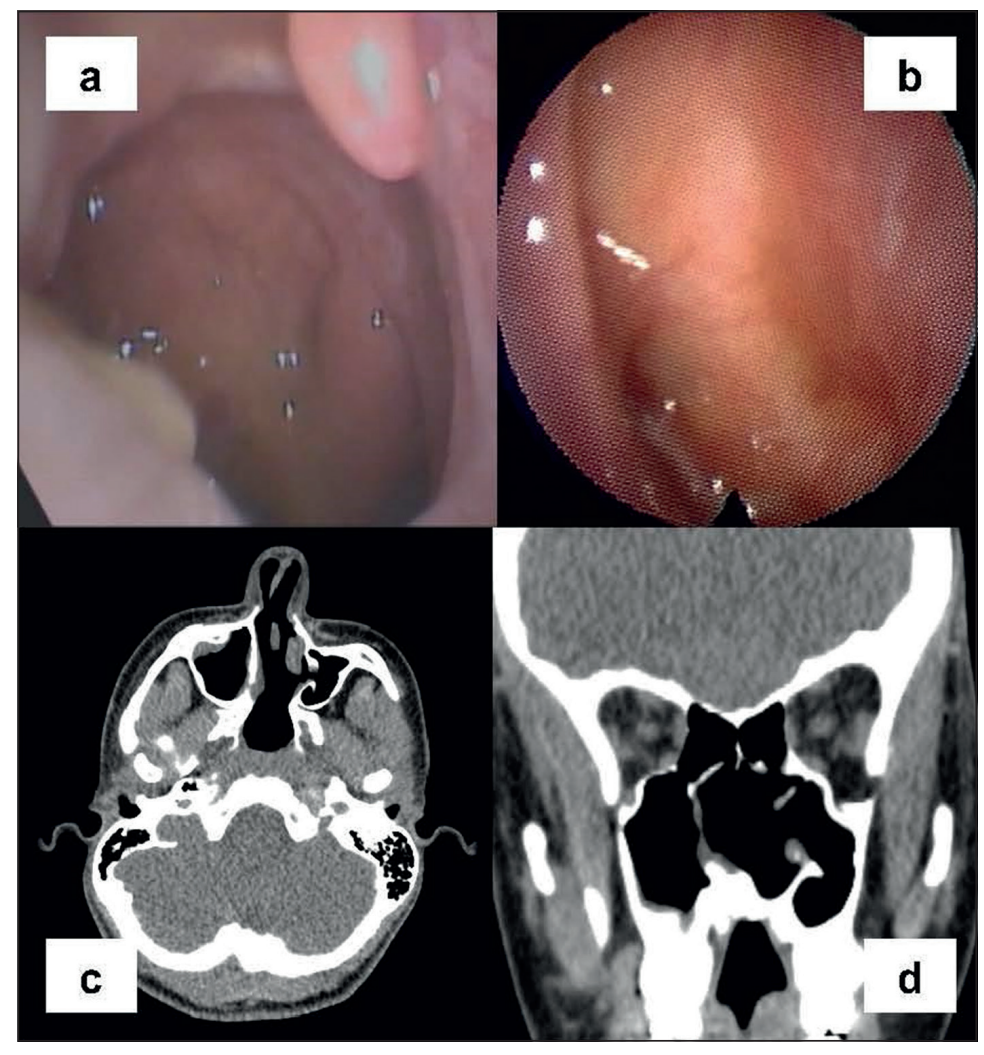

Figura 2. Imagen posoperatoria, a: neocoana desde fosa nasal derecha; b: meatotomía media permeable visualizando pared posterior de seno maxilar izquierdo; c: TC corte axial: septectomía posterior junto con perforación septal anterior; seno maxilar izquierdo hipoplásico sin secreciones y neocoana permeable; d: TC corte coronal: septectomía posterior con neocoana y meatotomía media izquierda permeables, seno maxilar izquierdo y ambas fosas nasales libres de secreciones. Puede observarse la asimetría del suelo de la fosa nasal izquierda con respecto a la derecha. entre un 10\%-50\% de los casos, siendo el más frecuente el síndrome de CHARGE. Existe predominio femenino $2: 1$, y su diagnóstico puede pasar desapercibido hasta incluso años después del nacimiento, no siendo así en los casos bilaterales, porque los recién nacidos son respiradores nasales obligados y en estos casos el diagnóstico se realiza en la propia sala del paritorio, siendo necesaria una intubación orotraqueal para poder tener una vía aérea permeable y realizar el diagnóstico inicial a través de una imposibilidad de sondaje nasal, una endoscopia nasal o un TC de senos paranasales. Aunque este es un dogma que no se pone en duda, desde 1991 se han publicado varios casos aislados diagnosticados en la adolescencia o en la edad adulta ${ }^{4}$, porque a veces esta malformación no siempre produce un distrés respiratorio agudo en el recién nacido ${ }^{1}$. El retraso en el diagnóstico se vuelve con los años menos probable, pero se han publicado casos en la segunda, tercera o incluso la quinta década de la vida ${ }^{5}$. No hay que confundir estos casos con la atresia de coanas adquirida ${ }^{6}$, en la que fácilmente por la historia clínica, la anamnesis y la exploración se puede establecer el diagnóstico.

El abordaje endoscópico nasosinusal es actualmente la técnica de elección para su tratamiento ${ }^{7}$, aunque la necesidad de realizar taponamiento nasal, uso de mitomicina $\mathrm{C}$ o colocación de tutores en el período posoperatorio continúa siendo un tema controvertido ${ }^{8}$. En el caso presentado, la cirugía se podría haber realizado en dos tiempos: el primero para corregir la desviación septal obstructiva y drenar el seno maxilar izquierdo mediante una antrostomía maxilar y el segundo para corregir la atresia de coana bilateral. Hipotéticamente se podría haber prevenido la devascularización de la mucosa septal y el posterior desarrollo de la perforación septal.

Existen 17 casos de atresia de coana bilateral diagnosticados en adultos publicados en la actualidad en la literatura anglosajona, pero sólo uno con malformación asociada (picno- 
disostosis $)^{9}$ y otro con hipoplasia de senos paranasales con hipogammaglobulinemia ${ }^{10}$, pero ninguno asociando cirugía de malformación craneofacial (labio leporino, síndrome de seno silente y desviación septal asociadas).

\section{Conclusión}

La atresia de coana unilateral normalmente se presenta desde el nacimiento como un distress respiratorio y dificultad para la alimentación, pero a veces puede pasar inadvertida durante la infancia y ser diagnosticada más tarde. Por el contrario, la atresia bilateral siempre se ha considerado como una urgencia absoluta en el posparto inmediato, por ser incompatible con la vida al ser los recién nacidos respiradores nasales obligados. En los últimos 30 años, 17 pacientes han sido diagnosticados e intervenidos en la adolescencia o la edad adulta, poniendo esta premisa en cuestión.

Aunque el abordaje transpalatal se ha utilizado durante muchos años, hoy en día la cirugía endoscópica es el gold standard para el tratamiento, continúa habiendo discusión sobre la necesidad de utilizar mitomicina C, taponamientos nasales o stents. El caso que presentamos es el primero que requiere una corrección quirúrgica de diferentes áreas anatómicas próximas entre sí, por lo que se plantea un problema de elección en el orden de corrección de las mismas. Este problema seguirá siendo tema de controversia hasta que los resultados de más casos sean conocidos en el futuro.

\section{Bibliografía}

1. Candan S, Mizrak SJ, Karagöz M, Muhtar H, Gümele HR. Bilateral congenital choanal atresia at age 16. An interesting case. J Otolaryngol. 1991; 20(6): 433-4.

2. Canovas DL, Carbonell J. Anales ORL Iber-amer. 1994;5:487-96.

3. Latifi AA, Hens E, Kakaria AK. Bilateral congenital choanal atresia presenting at age twenty two years: an unusual case report. Indian J Otolaryngol Head Neck Surg. 1999;51(1):59-61. doi: 10.1007/BF02996849.

4. Verma RK, Lokesh P, Panda NK. Congenital bilateral adult choanal atresia undiagnosed until the second decade: How we did it. Allergy Rhinol (Providence). 2016;7(2):82-84. doi: 10.2500/ar.2016.7.0155.

5. Yasar H, Ozkul MH. Bilateral congenital choanal atresia in a 51-year-old woman. Am J Rhinol. 2007;21(6):716-718. doi: 10.2500/ajr.2007.21.3100.

6. Nazar R, Naser A, Fullá J. Atresia de coanas en la edad adulta. Rev Otorrinolaringol Cir Cabeza Cuello. 2008; 68:178-84. doi: 10.4067/S0178-48162008000200010.

7. Chaudhary N, Jain A, Kapoor R, Motwani G. Bilateral complete choanal atresia in an adult woman: Managed with nasal endoscopes. J Indian Med Assoc. 2010;108(2):109-10.

8. Aksoy F, Demirhan H, Yildirim YS, Ozturan O. Bilateral choanal atresia in an adult - management with mitomycin C and without stents: a case report. Cases J. 2009;2:9307. doi: 10.1186/1757-16262-9307.

9. Durmaz CD, Ta V, Kocaay P, et al. Bilateral choanal atresia in an adult woman with pycnodysostosis. Congenit Anom (Kyoto). 2017;57(3):91-92. doi: 10.1111/cga.12204.

10. El-Sawy H, Siddiq MA, Anbarasu A. Bilateral choanal atresia and paranasal sinus hypoplasia in an adult patient with hypogammaglobulinaemia. Eur Arch Otorhinolaryngol. 2006;263(12):1136-1138. doi: 10.1007/s00405-006-0108-9. 